\title{
Concise Commentary: The Clot Thickens-Why Pylephlebitis Is One of the Most Feared Complications of Intra-abdominal Sepsis
}

\author{
Jonathan G. Stine ${ }^{1}$ \\ Published online: 2 January 2019 \\ ○) Springer Science+Business Media, LLC, part of Springer Nature 2019
}

Pylephlebitis is initiated by thrombophlebitis of the venules draining the area of acute infection. Subsequent migratory thrombophlebitis develops with extension into the larger veins of the portal system, which can include the portal vein (right, left or main) with or without extension into either the splenic or the superior mesenteric vein (SMV), the latter of which can occur in upwards of $40 \%$ of cases [1].

In this issue of the Digestive Diseases and Sciences, Zardi et al. [2] report a case of septic pylephlebitis in a 41-yearold patient with well-controlled human immunodeficiency virus (HIV) and multiple infections including intra-abdominal cholangitis. The patient was treated both with long-term antibiotics with infection resolution and concurrent lifelong anticoagulation (AC), although only partial recanalization was achieved with medical therapy. The patient continued with chronic right intrahepatic portal vein thrombosis (PVT) without portal hypertension. Overall, this is an interesting report that highlights a rare condition of infective suppurative thrombosis of the portal vein in an established patient population; this phenomenon is well described in patients with HIV. It provides a reminder to the busy clinician that pylephlebitis should be considered in all patients with intra-abdominal sepsis of any origin when accompanied by abdominal pain and abnormal liver-associated enzymes.

In general, thrombosis of the SMV is problematic since it may lead to bowel ischemia and intestinal infarction requiring emergent surgical exploration and bowel resection [3]. Testing for a hypercoagulable state should be pursued since it is present in $\sim 40 \%$ of cases of pylephlebitis [4]. Inherited or acquired thrombophilia appears to be less common in pylephlebitis than in cirrhosis patients who develop chronic

Jonathan G. Stine

jstine@ pennstatehealth.psu.edu

1 Division of Gastroenterology and Hepatology, Department of Medicine, and Department of Public Health Sciences, The Pennsylvania State University Milton S. Hershey Medical Center, Hershey, PA, USA non-tumoral PVT. To date, only lupus anticoagulant positivity is associated with pylephlebitis [4]; no cases of the more commonly identified thrombophilias in cirrhosis including factor V Leiden, prothrombin gene G20210A mutation or Janus kinase (JAK)-2 mutation have been reported [5].

The contribution of AC therapy toward the treatment of pylephlebitis remains controversial. AC is required when thrombophilia is present or if SMV involvement is documented. Beyond this, additional reports suggest that AC may increase the rate of portal vein recanalization, which in turn may attenuate development of portal hypertension. Despite its benefits, no consensus exists regarding the optimal AC regimen. The most extensive experience has been reported with vitamin $\mathrm{K}$ antagonist (VKA) use although VKAs require careful monitoring and may be limited by a narrow therapeutic window if an abnormal baseline prothrombin time-international normalized ratio (PT-INR) is present. Low-molecular weight heparin (LMWH) is also limited in practicality but can be prescribed. While direct-acting oral anticoagulants are gaining increasing popularity in Child-Turcotte-Pugh class A and B cirrhosis patients with PVT, less experience with treatment of acute pylephlebitis exists, where only rivaroxaban has been prescribed in this setting according to published reports. The optimal duration of AC therapy for pylephlebitis also remains unknown.

In conclusion, pylephlebitis is a rare complication of intra-abdominal sepsis requiring prompt diagnosis and initiation of medical treatment with antibiotics. AC in pylephlebitis remains controversial although there is a growing body of evidence that AC may decrease morbidity and mortality through the promotion of portal vein recanalization, thus preventing chronic portal hypertensive complications.

\section{References}

1. Kanellopoulou T, Alexopoulou A, Theodossiades G, Koskinas J, Archimandritis AJ. Pylephlebitis: an overview of non-cirrhotic 
cases and factors related to outcome. Scand J Infect Dis. 2010;42:804-811.

2. Zardi et al. Pylephlebitis as a complication of cholangitis and sepsis in a HIV positive patient. A case report. Dig Dis Sci. (Epub ahead of print). https://doi.org/10.1007/s10620-018-5391-y.

3. Amitrano L, Guardascione MA, Brancaccio V, et al. Risk factors and clinical presentation of portal vein thrombosis in patients with liver cirrhosis. J Hepatol. 2004;40:736-741.
4. Pradka SP, Trankiem CT, Ricotta JJ. Pylephlebitis and acute mesenteric ischemia in a young man with inherited thrombophilia and suspected foodborne illness. J Vasc Surg. 2012;55:1769-1772.

5. Saugel B, Lee M, Feichtinger S, Hapfelmeier A, Schmid RM, Siveke JT. Thrombophilic factor analysis in cirrhotic patients with portal vein thrombosis. J Thromb Thrombolysis. 2015;40:54-60. 